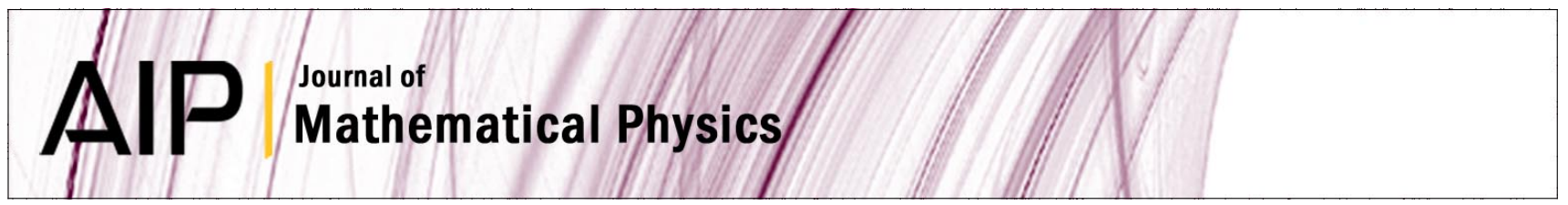

\title{
Effects of Marangoni numbers on thermocapillary drop migration: Constant for quasi-steady state?
}

Zuo-Bing Wu and Wen-Rui Hu

Citation: J. Math. Phys. 54, 023102 (2013); doi: 10.1063/1.4792476

View online: http://dx.doi.org/10.1063/1.4792476

View Table of Contents: http://jmp.aip.org/resource/1/JMAPAQ/v54/i2

Published by the American Institute of Physics.

\section{Related Articles}

Bubble motion and size variation during thermal migration with phase change Phys. Fluids 25, 013302 (2013)

Numerical simulations on thermocapillary migrations of nondeformable droplets with large Marangoni numbers Phys. Fluids 24, 092101 (2012)

Effect of slippage on the thermocapillary migration of a small droplet Biomicrofluidics 6, 012809 (2012)

Thermocapillary instabilities in an evaporating drop deposited onto a heated substrate Phys. Fluids 24, 032103 (2012)

Thermocapillary motion of a slender viscous droplet in a channel Phys. Fluids 24, 022102 (2012)

\section{Additional information on J. Math. Phys.}

Journal Homepage: http://jmp.aip.org/

Journal Information: http://jmp.aip.org/about/about_the_journal

Top downloads: http://jmp.aip.org/features/most_downloaded

Information for Authors: http://jmp.aip.org/authors

\section{ADVERTISEMENT}

The most comprehensive support for physics in any mathematical software package World-leading tools for performing calculations in theoretical physics

Your work in Maple matches how you would write the problems and solutions by hand

- State-of-the-art environment for algebraic computations in physics

The only system with the ability to handle a wide range of physics computations as well as pencil-and-paper style input and textbook-quality display of results

- Access to Maple's full mathematical power, programming language, visualization routines, and document creation tools 


\title{
Effects of Marangoni numbers on thermocapillary drop migration: Constant for quasi-steady state?
}

\author{
Zuo-Bing $\mathrm{Wu}^{1, a)}$ and Wen-Rui $\mathrm{Hu}^{2}$ \\ ${ }^{1}$ State Key Laboratory of Nonlinear Mechanics, Institute of Mechanics, \\ Chinese Academy of Sciences, Beijing 100190, China \\ ${ }^{2}$ National Microgravity Laboratory, Institute of Mechanics, \\ Chinese Academy of Sciences, Beijing 100190, China
}

(Received 10 May 2012; accepted 31 January 2013; published online 22 February 2013)

\begin{abstract}
The overall steady-state energy balance with two phases in a flow domain requires that the change in energy of the domain is equal to the difference between the total energy entering the domain and that leaving the domain. From the condition, the integral thermal flux across the surface is studied for a steady thermocapillary drop migration in a flow field with uniform temperature gradient at small and large Marangoni (Reynolds) numbers. The drop is assumed to have only a slight axisymmetric deformation from a sphere. It is identified that a conservative/nonconservative integral thermal flux across the surface in the steady thermocapillary drop migration at small/large Marangoni (Reynolds) numbers. The conservative flux confirms the assumption of quasi-steady state in the thermocapillary drop migration at small Marangoni (Reynolds) numbers. The nonconservative flux may well result from the invalid assumption of quasi-steady state, which indicates that the thermocapillary drop migration at large Marangoni (Reynolds) numbers cannot reach steady state and is thus a unsteady process. (C) 2013 American Institute of Physics. [http://dx.doi.org/10.1063/1.4792476]
\end{abstract}

\section{INTRODUCTION}

The motion of a drop or bubble in the microgravity environment embedded in an immiscible mother liquid with a uniform temperature gradient is termed as thermocapillary migration of the drop or bubble, which is a very interesting topic for both fundamental theory and engineering application. ${ }^{1}$ Young et al. (YGB) carried out an initial study in this area, called as YGB model, ${ }^{2}$ and gave an analytical prediction on its migration speed in the limit case of zero Reynolds $(R e)$ and zero Marangoni $(M a)$ numbers, and a series of theoretical analyses, numerical simulations and experimental investigations on this subject were carried out ever since. Subramanian ${ }^{3}$ and Crespo et $a l .{ }^{4}$ extended the YGB results to small $M a$ numbers and obtained analytical results in series expansion of $M a$ numbers. With consideration of thermal boundary layer, the analytical results for migration speed of a bubble at large $M a(R e)$ numbers ${ }^{5,6}$ agree well with the corresponding results of steady state numerical simulations ${ }^{4,7,8}$ and experimental studies. ${ }^{9}$

Although the thermocapillary bubble migration processes are understood very well, the behavior of thermocapillary drop migration appears rather complicated due to the transfer of momentum and energy though the interface of two-phase fluids. For the migration of a drop, on the one hand, the experimental result of the migration speed at small drops $11 \pm 1.5 \mu \mathrm{m}$ in diameter obtained by Braun et al. ${ }^{10}$ agrees with the YGB model. On the other hand, another experiment for larger drops with diameters ranging from 0.69 to $2.38 \mathrm{~mm}$ performed by Wozniak ${ }^{11}$ shows that the migration velocities are smaller than those given by the YGB linear prediction. Afterward more attention has been paid to thermocapillary drop migration for large $M a(R e)$ numbers. Hadland

\footnotetext{
a) Author to whom correspondence should be addressed. Electronic mail: wuzb@lnm.imech.ac.cn. Tel.: + 86-10-82543955. Fax: + 86-10-82543977.
} 
et al. ${ }^{9}$ carried out experiments based on Fluorinert liquid FC-75 and 10cst silicone oil as the drop phase and continuous phase during the NASA Space Shuttle mission with the maximal $M a(R e)$ number up to 3300(49.1). It was shown that the drop migration speed nondimensionalized by the YGB velocity decreased as the $M a$ number increased and the global migration process exhibited an unsteady nature. To further observe the variation trend of drop migration velocity with increasing Ma number, Xie et al. ${ }^{12}$ adopted Fluorinert liquid FC-75 and 5cst silicone oil as the drop and the continuous phases, respectively, and performed experiments in the Chinese spacecraft ShenZhou-4. The experimental investigation was completed for several ranges of large $M a(R e)$ numbers, where the drop did not reach the steady state in the migration process, and the maximal $M a(R e)$ number reached 5525(302.6). It was also observed that the non-dimensional drop migration velocity decreased as the $M a$ numbers increased. However, from the theoretical analysis for the large $M a(R e)$ numbers, it was reported ${ }^{13}$ that the migration speed of a drop increased with increasing $M a$ number, which is in qualitative agreement with the corresponding numerical simulation. ${ }^{14}$ Both the theoretical analysis and numerical simulation are based on the assumptions of quasi-steady state and nondeformation of the drop. The above qualitative difference between experimental observations and theoretical/numerical results may result from the quasi-steady state or non-deformation assumptions of the drop in the model. Moreover, Herrmann et al. ${ }^{15}$ and Wu et al. ${ }^{16}$ adopted respectively the numerical methods to investigate the thermocapillary motion of deformable and non-deformable drops and indicated that the assumption of quasi-steady state was not valid for large $M a$ numbers. Therefore, the thermocapillary drop migration at large $M a(R e)$ numbers is still a topic to be further studied with emphasis laid on its physical mechanism.

To address the discrepancies between experimental and theoretical/numerical results, in this paper, our effects are focused on the assumption of quasi-steady state in the process of thermocapillary drop migration. The drop may have only a slight axisymmetric deformation from a sphere. By using the asymptotic expansion method, we investigate the continuity of integral thermal flux across the surface based on the overall steady-state energy balance in the flow domain, and analyze the existence of quasi-steady migration of the drop at zero, small and large $M a(R e)$ numbers.

\section{MODELS AND QUASI-STEADY STATE ASSUMPTION}

Consider the thermocapillary migration of a spherical drop of radius $R_{0}$, density $\gamma \rho$, dynamic viscosity $\alpha \mu$, thermal conductivity $\beta k$, and thermal diffusivity $\lambda \kappa$ in a continuous phase fluid of infinite extent with density $\rho$, dynamic viscosity $\mu$, thermal conductivity $k$, and thermal diffusivity $\kappa$ under a uniform temperature gradient $G$. The change rate of the interfacial tension between the drop and the continuous phase fluid with temperature is denoted by $\sigma_{T}$. Axisymmetric energy equations for the continuous phase and for the fluid in the drop in a laboratory coordinate system denoted by a bar are written as follows:

$$
\begin{aligned}
& \frac{\partial \bar{T}}{\partial t}+\overline{\mathbf{v}} \bar{\nabla} \bar{T}=\kappa \bar{\Delta} \bar{T}, \\
& \frac{\partial \bar{T}^{\prime}}{\partial t}+\overline{\mathbf{v}}^{\prime} \bar{\nabla} \bar{T}^{\prime}=\lambda \kappa \bar{\Delta} \bar{T}^{\prime},
\end{aligned}
$$

where $\overline{\mathbf{v}}$ and $\bar{T}$ are velocity and temperature, and a prime denotes quantities inside the drop. The solutions of Eq. (1) have to satisfy the boundary conditions at infinity

$$
\bar{T}_{\infty} \rightarrow T_{0}+G \bar{z},
$$

where $T_{0}$ is the undisturbed temperature of the continuous phase and the boundary conditions at the interface $\left(\bar{r}_{b}, \bar{z}_{b}\right)$ of the two fluids

$$
\begin{aligned}
& \bar{T}\left(\bar{r}_{b}, \bar{z}_{b}, t\right)=\bar{T}^{\prime}\left(\bar{r}_{b}, \bar{z}_{b}, t\right), \\
& \frac{\partial \bar{T}}{\partial n}\left(\bar{r}_{b}, \bar{z}_{b}, t\right)=\beta \frac{\partial \bar{T}^{\prime}}{\partial n}\left(\bar{r}_{b}, \bar{z}_{b}, t\right),
\end{aligned}
$$

where $\mathbf{n}$ is a unit vector normal to the interface. In what follows, the undisturbed temperature $T_{0}$ is reduced for simplicity. 
In general, the surface tension decreases with the increasing of the local temperature. For a temperature field with its gradient in the $\bar{z}$ direction, the generated surface tension force is a net force along the surface and the droplet starts to move towards the warm side under the action of net force. When the net force acting on the drop at the flow direction is zero, the thermocapillary drop migration reaches a stable process. However, due to the variation of physical parameters with the ambient temperature, the migration process may not reach stable state. Only when the migration is sufficiently slow that the order of relevant time scale for the transport process to generate stable velocity and temperature fields is smaller than that for the drop to move an appreciable distance, the assumption of the quasi-steady state is valid. It means that after experiencing an initial unstable migration process, the drop migration may reach a steady state at the time $t_{0}$ and the position $\mathbf{r}_{0}$ $=z_{0} \mathbf{k}$, i.e., migrating with a constant drop migration speed $V_{\infty}$. Using the coordinate transformation from the laboratory coordinate system to a coordinate system moving with the drop velocity $V_{\infty}$,

$$
\begin{aligned}
& \overline{\mathbf{r}}=\mathbf{r}+\mathbf{r}_{0}+V_{\infty}\left(t-t_{0}\right) \mathbf{k}, \quad \overline{\mathbf{v}}(\overline{\mathbf{r}}, t)=\mathbf{v}(\mathbf{r})+V_{\infty} \mathbf{k}, \quad \bar{T}(\overline{\mathbf{r}}, t)=T(\mathbf{r})+G\left[z_{0}+V_{\infty}\left(t-t_{0}\right)\right], \\
& \overline{\mathbf{v}}^{\prime}(\overline{\mathbf{r}}, t)=\mathbf{v}^{\prime}(\mathbf{r})+V_{\infty} \mathbf{k}, \quad \bar{T}^{\prime}(\overline{\mathbf{r}}, t)=T^{\prime}(\mathbf{r})+G\left[z_{0}+V_{\infty}\left(t-t_{0}\right)\right],
\end{aligned}
$$

the problem (1) can be formulated as

$$
\begin{aligned}
& G V_{\infty}+\mathbf{v} \nabla T=\kappa \Delta T, \\
& G V_{\infty}+\mathbf{v}^{\prime} \nabla T^{\prime}=\lambda \kappa \Delta T^{\prime} .
\end{aligned}
$$

The details of the transformation are given in the Appendix. By taking the radius of the drop $R_{0}$, the velocity $v_{0}=-\sigma_{T} G R_{0} / \mu$ and $G R_{0}$ as the reference quantities to make the coordinates, velocity and temperature dimensionless, energy equations (5) combined with the continuous equations can be written in the following dimensionless form in a spherical coordinate system $(r, \theta)$ :

$$
\begin{aligned}
V_{\infty}+\nabla \cdot(\mathbf{v} T) & =\frac{1}{M a} \Delta T, \\
V_{\infty}+\nabla \cdot\left(\mathbf{v}^{\prime} T^{\prime}\right) & =\frac{\lambda}{M a} \Delta T^{\prime},
\end{aligned}
$$

where $\mathbf{v}=(u, v)$ and Marangoni number is defined as

$$
M a=\frac{v_{0} R_{0}}{\kappa} .
$$

By using the transformation (4), the boundary conditions (2) and (3) can be respectively written in the form of dimensionless as follows:

$$
T \rightarrow r \cos \theta, \text { as } r \rightarrow \infty
$$

at places far away from the drop and

$$
\begin{aligned}
T\left(r_{0}, \theta\right) & =T^{\prime}\left(r_{0}, \theta\right), \\
\frac{\partial T}{\partial n}\left(r_{0}, \theta\right) & =\beta \frac{\partial T^{\prime}}{\partial n}\left(r_{0}, \theta\right)
\end{aligned}
$$

at the interface of the two fluids. Thus, once the drop migration reaches a steady state, the above problem in the laboratory coordinate system can be described by the steady energy equations (6) and (7) with the boundary conditions (9)-(11) in the coordinate system moving with the drop velocity. This implies the overall steady-state energy balance with two phases in the flow domain in the co-moving frame of reference.

\section{INTEGRAL THERMAL FLUX ACROSS THE DROP SURFACE UNDER THE QUASI-STEADY STATE ASSUMPTION}

In general, for a two-phase flow, as is the case in the present problem, if the quasi-steady state assumption is valid, the solutions of the problem not only satisfy the differential energy 
equations with boundary conditions, but also preserve the overall steady-state energy balance with two phases in the flow domain under integral boundary conditions. However, if the quasi-steady state assumption is invalid, the overall steady-state energy with two phases in the flow domain under integral boundary conditions is not balanced. This means the solutions of the problem also cannot satisfy the differential energy equations with boundary conditions. Thus, to confirm whether the thermocapillary drop migrations at different $M a(R e)$ numbers are always in the quasi-steady state processes, we may analyze the overall steady-state energy of two phases in the flow domain in the co-moving frame of reference under integral boundary conditions.

For the thermocapillary drop migration, to get the overall steady-state energy transport of two phases in the flow domain in the co-moving frame of reference, we have to integrate Eqs. (6) and (7) in the continuous phase domain $\left(r \in\left[r_{0}, r_{\infty}\right], \theta \in[0, \pi]\right)$ and within the drop region $\left(r \in\left[0, r_{0}\right]\right.$, $\theta \in[0, \pi])$ as

$$
\begin{aligned}
& \int_{r_{0}}^{\infty} \int_{0}^{\pi} V_{\infty} d V+\int_{r_{0}}^{\infty} \int_{0}^{\pi} \nabla \cdot(\mathbf{v} T) d V=\frac{1}{M a} \int_{r_{0}}^{\infty} \int_{0}^{\pi} \Delta T d V, \\
& \int_{0}^{r_{0}} \int_{0}^{\pi} V_{\infty} d V+\int_{0}^{r_{0}} \int_{0}^{\pi} \nabla \cdot\left(\mathbf{v}^{\prime} T^{\prime}\right) d V=\frac{\lambda}{M a} \int_{0}^{r_{0}} \int_{0}^{\pi} \Delta T^{\prime} d V,
\end{aligned}
$$

and Eq. (10) and Eq. (11) at the drop surface $\left(r=r_{0}, \theta \in[0, \pi]\right)$ as

$$
\begin{gathered}
\int_{0}^{\pi} T\left(r_{0}, \theta\right) d S=\int_{0}^{\pi} T^{\prime}\left(r_{0}, \theta\right) d S, \\
\int_{0}^{\pi} \frac{\partial T}{\partial n}\left(r_{0}, \theta\right) d S=\beta \int_{0}^{\pi} \frac{\partial T^{\prime}}{\partial n}\left(r_{0}, \theta\right) d S,
\end{gathered}
$$

where $d V=r^{2} \sin \theta d r d \theta$ and $d S=r^{2} \sin \theta d \theta$. And then transforming the volume integration of Eq. (12) and Eq. (13) in the flow domains to the surface integration over the droplet surface and the surface at infinity in terms of the Gaussian formula, we have

$$
\frac{2 V_{\infty}}{3}\left(r_{\infty}^{3}-\frac{1}{2} \int_{0}^{\pi} r_{0}^{3} \sin \theta d \theta\right)+\left.\oint(u T)\right|_{r_{\infty}} d S-\left.\oint(u T)\right|_{r_{0}} d S=\frac{1}{M a}\left(\left.\oint \frac{\partial T}{\partial n}\right|_{r_{\infty}} d S-\left.\oint \frac{\partial T}{\partial n}\right|_{r_{0}} d S\right)
$$

and

$$
\frac{V_{\infty}}{3} \int_{0}^{\pi} r_{0}^{3} \sin \theta d \theta+\left.\oint\left(u^{\prime} T^{\prime}\right)\right|_{r_{0}} d S=\left.\frac{\lambda}{M a} \oint \frac{\partial T^{\prime}}{\partial n}\right|_{r_{0}} d S .
$$

Using the zero normal velocity boundary condition at the interface, we can derive

$$
\begin{aligned}
\left.\int_{0}^{\pi} \frac{\partial T}{\partial n}\right|_{r_{0}} r_{0}^{2} \sin \theta d \theta= & \left.r_{\infty}^{2} \int_{0}^{\pi} \frac{\partial T}{\partial r}\right|_{r_{\infty}} \sin \theta d \theta-\left.\operatorname{Mar}_{\infty}^{2} \int_{0}^{\pi}(u T)\right|_{r_{\infty}} \sin \theta d \theta \\
& -\frac{2 V_{\infty} M a}{3}\left(r_{\infty}^{3}-\frac{1}{2} \int_{0}^{\pi} r_{0}^{3} \sin \theta d \theta\right)
\end{aligned}
$$

and

$$
\left.\int_{0}^{\pi} \frac{\partial T^{\prime}}{\partial n}\right|_{r_{0}} r_{0}^{2} \sin \theta d \theta=\frac{V_{\infty} M a}{3 \lambda} \int_{0}^{\pi} r_{0}^{3} \sin \theta d \theta,
$$

where the outer normal vector at infinity is the radial coordinate axis. Thus, Eq. (18) and Eq. (19) display integral thermal fluxes across the drop surface obtained from the overall energy transport. We assume that the drop has only a slight axisymmetric deformation from a sphere

$$
r_{0}=1+f(\theta), \quad f \ll 1 .
$$

For this case, Eq. (18) and Eq. (19) may be written as

$$
\begin{aligned}
\left.\int_{0}^{\pi} \frac{\partial T}{\partial n}\right|_{r_{0}} r_{0}^{2} \sin \theta d \theta= & \left.r_{\infty}^{2} \int_{0}^{\pi} \frac{\partial T}{\partial r}\right|_{r_{\infty}} \sin \theta d \theta-\left.\operatorname{Mar}_{\infty}^{2} \int_{0}^{\pi}(u T)\right|_{r_{\infty}} \sin \theta d \theta \\
& -\frac{2 V_{\infty} M a}{3}\left[r_{\infty}^{3}-1-\frac{3}{2} \int_{0}^{\pi} f \sin \theta d \theta+O\left(f^{2}\right)\right]
\end{aligned}
$$


and

$$
\left.\int_{0}^{\pi} \frac{\partial T^{\prime}}{\partial n}\right|_{r_{0}} r_{0}^{2} \sin \theta d \theta=\frac{V_{\infty} M a}{3 \lambda}\left[2+3 \int_{0}^{\pi} f \sin \theta d \theta+O\left(f^{2}\right)\right] .
$$

To next-to-leading (first) order in $f$, Eq. (21) and Eq. (22) may finally be written as

$$
\begin{aligned}
\left.\int_{0}^{\pi} \frac{\partial T}{\partial n}\right|_{r_{0}} r_{0}^{2} \sin \theta d \theta= & \left.r_{\infty}^{2} \int_{0}^{\pi} \frac{\partial T}{\partial r}\right|_{r_{\infty}} \sin \theta d \theta-\left.M a r_{\infty}^{2} \int_{0}^{\pi}(u T)\right|_{r_{\infty}} \sin \theta d \theta \\
& -\frac{2 V_{\infty} M a}{3}\left(r_{\infty}^{3}-1-\frac{3}{2} \int_{0}^{\pi} f \sin \theta d \theta\right)
\end{aligned}
$$

and

$$
\left.\int_{0}^{\pi} \frac{\partial T^{\prime}}{\partial n}\right|_{r_{0}} r_{0}^{2} \sin \theta d \theta=\frac{V_{\infty} M a}{3 \lambda}\left(2+3 \int_{0}^{\pi} f \sin \theta d \theta\right)
$$

From Eq. (14) and Eq. (15), we have integral boundary conditions across the drop surface

$$
\begin{gathered}
\left.\int_{0}^{\pi} T\right|_{r_{0}} r_{0}^{2} \sin \theta d \theta=\left.\int_{0}^{\pi} T^{\prime}\right|_{r_{0}} r_{0}^{2} \sin \theta d \theta \\
\left.\int_{0}^{\pi} \frac{\partial T}{\partial n}\right|_{r_{0}} r_{0}^{2} \sin \theta d \theta=\left.\beta \int_{0}^{\pi} \frac{\partial T^{\prime}}{\partial n}\right|_{r_{0}} r_{0}^{2} \sin \theta d \theta .
\end{gathered}
$$

Thus, for a quasi-steady state thermocapillary migration of the drop with the slight deformation from the sphere, the overall steady-state energy balance of two phases in the flow domain in the co-moving frame of reference requires that the integral thermal fluxes (23) and (24) at the drop surface obtained from the overall energy transport are self-consistent with the integral boundary condition (26). In the following, we will investigate the self-consistency for the different $M a(R e)$ numbers.

\section{A. Conservative integral thermal flux across the drop surface at zero $M a(R e)$ numbers}

In the case of zero $\operatorname{Re}\left(\operatorname{Re}=\frac{v_{0} R_{0}}{v}\right)$ and zero $M a$ numbers, i.e., the YGB model, scaled velocity and temperature fields of the continuous phase and within the drop in Eq. (6) and Eq. (7) may be described $^{2,17}$ as

$$
\begin{aligned}
& u=-V_{\infty} \cos \theta\left(1-\frac{1}{r^{3}}\right), \\
& v=V_{\infty} \sin \theta\left(1+\frac{1}{2 r^{3}}\right), \\
& T=\left(r+\frac{1-\beta}{2+\beta} \frac{1}{r^{2}}\right) \cos \theta
\end{aligned}
$$

and

$$
\begin{aligned}
u^{\prime} & =\frac{3}{2} V_{\infty} \cos \theta\left(1-r^{2}\right), \\
v^{\prime} & =-\frac{3}{2} V_{\infty} \sin \theta\left(1-2 r^{2}\right), \\
T^{\prime} & =\frac{3}{2+\beta} r \cos \theta
\end{aligned}
$$

Since $M a=0$, using the temperature field in (27), we can derive the following equality from Eq. (23) and Eq. (24)

$$
\left.\int_{0}^{\pi} \frac{\partial T}{\partial n}\right|_{r_{0}} r_{0}^{2} \sin \theta d \theta=\left.\beta \int_{0}^{\pi} \frac{\partial T^{\prime}}{\partial n}\right|_{r_{0}} r_{0}^{2} \sin \theta d \theta=0 .
$$

Thus, under the quasi-steady state assumption, the integral thermal flux across the drop surface at zero $R e$ and zero $M a$ numbers is conservative, which corresponds to the integral thermal flux boundary condition (26). This implies the overall steady-state energy balance of two phases in the flow domain in the co-moving frame of reference. The quasi-steady state assumption is valid. 


\section{B. Conservative integral thermal flux across the drop surface at small $M a(R e)$ numbers}

For small Re number, the velocity fields in Eq. (6) and Eq. (7) may be described by the creeping flow. The general solutions of the scaled flow field in the continuous phase and within the drop are given by ${ }^{18,19}$ as

$$
\begin{aligned}
& u=-V_{\infty} \cos \theta\left(1-\frac{1}{r^{3}}\right)-\left(1-\frac{1}{r^{2}}\right) \sum_{n=3}^{\infty} D_{n} r^{-n+1} P_{n-1}(\cos \theta), \\
& v=V_{\infty} \sin \theta\left(1+\frac{1}{2 r^{3}}\right)+\sum_{n=3}^{\infty} D_{n}\left(\frac{-n+3}{r^{n-1}}-\frac{-n+1}{r^{n+1}}\right) C_{n}^{-1 / 2}(\cos \theta) / \sin \theta,
\end{aligned}
$$

and

$$
\begin{aligned}
& u^{\prime}=\frac{3}{2} V_{\infty} \cos \theta\left(1-r^{2}\right)+\left(\frac{1}{r^{2}}-1\right) \sum_{n=3}^{\infty} D_{n} r^{n} P_{n-1}(\cos \theta), \\
& v^{\prime}=-\frac{3}{2} V_{\infty} \sin \theta\left(1-2 r^{2}\right)-\sum_{n=3}^{\infty} D_{n}\left[n r^{n-2}-(n+2) r^{n}\right] C_{n}^{-1 / 2}(\cos \theta) / \sin \theta,
\end{aligned}
$$

where $C_{n}^{-1 / 2}(\cos \theta)$ is the Gegenbauer polynomial of order $n$ and degree $-\frac{1}{2}, P_{n-1}(\cos \theta)$ is the Legendre polynomial of order $n . D_{n}$ is given as

$$
D_{n}=-\frac{n(n-1)}{4(1+\alpha)} \int_{0}^{\pi} C_{n}^{-1 / 2}(\cos \theta) \frac{\partial T}{\partial \theta} d \theta .
$$

And scaled temperature fields in the continuous phase and within the drop at the small Ma numbers are given in ${ }^{19}$ as

$$
\begin{aligned}
T= & \left(r+\frac{1-\beta}{2+\beta} \frac{1}{r^{2}}\right) \cos \theta \\
& +\frac{1}{3 \lambda(2+\beta)^{2}(2+3 \alpha)}\left[\frac{\delta_{1}}{r}+\frac{\delta_{2}}{r^{4}}+P_{2}(\cos \theta)\left(\frac{\delta_{3}}{r}+\frac{\delta_{4}}{r^{3}}+\frac{2 \delta_{2}}{r^{4}}\right)\right] \epsilon+O\left(\epsilon^{2}\right), \\
T^{\prime}= & \frac{3}{2+\beta} r \cos \theta \\
& +\frac{1}{\lambda(2+\beta)^{2}(2+3 \alpha)}\left[\delta_{1}^{\prime}+\delta_{2}^{\prime} r^{2}-\frac{3}{4} r^{4}+P_{2}(\cos \theta)\left(\delta_{4}^{\prime} r^{2}+\frac{3}{7} r^{4}\right)\right] \epsilon+O\left(\epsilon^{2}\right),
\end{aligned}
$$

where $\delta_{1}=2[\lambda(1-\beta)-\beta(2+\beta)], \delta_{2}=-\frac{\lambda}{2}(1-\beta), \delta_{3}=-\lambda(4-\beta), \delta_{4}=\frac{1}{7(3+2 \beta)}[7 \lambda(8$ $\left.\left.+5 \beta-4 \beta^{2}\right)-18 \beta\right], \delta_{1}^{\prime}=\frac{1}{12}\left[6 \lambda(1-\beta)-\left(8 \beta^{2}+20 \beta+17\right)\right], \delta_{2}^{\prime}=\frac{1}{6}(2 \beta+13), \delta_{4}^{\prime}=-\frac{1}{21(3+2 \beta)}$ $[7 \lambda(7-\beta)+9(3+4 \beta)]$ and the small parameter is $\epsilon=M a$. Then, using the temperature field in (33), we simplify Eq. (23) and Eq. (24) to

$$
\begin{aligned}
\left.\int_{0}^{\pi} \frac{\partial T}{\partial n}\right|_{r_{0}} r_{0}^{2} \sin \theta d \theta= & -\frac{2}{3 \lambda(2+\beta)^{2}(2+3 \alpha)}\left(\delta_{1}+\frac{4 \delta_{2}}{r_{\infty}^{3}}\right) \epsilon+\frac{2(1-\beta)}{3(2+\beta)}\left(1-\frac{1}{r_{\infty}^{3}}\right) V_{\infty} \epsilon \\
& +V_{\infty} \int_{0}^{\pi} f \sin \theta d \theta \epsilon+O\left(\epsilon^{2}\right) \\
\approx & -\frac{2 \delta_{1}}{3 \lambda(2+\beta)^{2}(2+3 \alpha)} \epsilon+\frac{2(1-\beta)}{3(2+\beta)} V_{\infty} \epsilon+V_{\infty} \int_{0}^{\pi} f \sin \theta d \theta \epsilon+O\left(\epsilon^{2}\right) \\
= & {\left[\frac{2 \beta}{3 \lambda} V_{\infty}^{0}+\frac{2(1-\beta)}{3(2+\beta)}\left(V_{\infty}-V_{\infty}^{0}\right)+V_{\infty} \int_{0}^{\pi} f \sin \theta d \theta\right] \epsilon+O\left(\epsilon^{2}\right) }
\end{aligned}
$$

and

$$
\left.\int_{0}^{\pi} \frac{\partial T^{\prime}}{\partial n}\right|_{r_{0}} r_{0}^{2} \sin \theta d \theta=\frac{V_{\infty}}{3 \lambda}\left(2+3 \int_{0}^{\pi} f \sin \theta d \theta\right) \epsilon,
$$

where $V_{\infty}^{0}=\frac{2}{(2+\beta)(2+3 \alpha)}$ is the migration velocity of the droplet at zero Re and zero $M a$ numbers. From Eq. (34) and Eq. (35), we have

$$
\begin{aligned}
\int_{0}^{\pi}\left(\left.\frac{\partial T}{\partial n}\right|_{r_{0}}-\left.\beta \frac{\partial T^{\prime}}{\partial n}\right|_{r_{0}}\right) r_{0}^{2} \sin \theta d \theta= & \frac{2}{3}\left(\frac{\beta}{\lambda}-\frac{1-\beta}{2+\beta}\right)\left(V_{\infty}^{0}-V_{\infty}\right) \epsilon \\
& +\left(1-\frac{\beta}{\lambda}\right) V_{\infty} \int_{0}^{\pi} f \sin \theta d \theta \epsilon+O\left(\epsilon^{2}\right) .
\end{aligned}
$$

Since $\epsilon \rightarrow 0\left(V_{\infty} \rightarrow V_{\infty}^{0}\right)$ and $f \ll 1$, we have

$$
\left.\left.\int_{0}^{\pi} \frac{\partial T}{\partial n}\right|_{r_{0}} r_{0}^{2} \sin \theta d \theta \approx \beta \int_{0}^{\pi} \frac{\partial T^{\prime}}{\partial n}\right|_{r_{0}} r_{0}^{2} \sin \theta d \theta .
$$


Thus, under the quasi-steady state assumption, the integral thermal flux across the drop surface at small $R e$ and small $M a$ numbers is conservative, which corresponds to the thermal flux boundary condition (26). This implies the overall steady-state energy balance of two phases in the flow domain in the co-moving frame of reference. The quasi-steady state assumption is valid.

\section{Nonconservative integral thermal flux across the drop surface at large $\mathrm{Ma}(R e)$ numbers}

For large Re number, the velocity fields in Eq. (6) and Eq. (7) can be described by potential flows and boundary layer flows. ${ }^{20}$ The scaled inviscid velocity field in the continuous phase and Hill's spherical vortex within the drop can be respectively written as

$$
\begin{aligned}
& u=-V_{\infty} \cos \theta\left(1-\frac{1}{r^{3}}\right), \\
& v=V_{\infty} \sin \theta\left(1+\frac{1}{2 r^{3}}\right)
\end{aligned}
$$

and

$$
\begin{aligned}
& u^{\prime}=\frac{3 V_{\infty}}{2} \cos \theta\left(1-r^{2}\right), \\
& v^{\prime}=-\frac{3 V_{\infty}}{2} \sin \theta\left(1-2 r^{2}\right) .
\end{aligned}
$$

Since Eq. (9) only gives the primary approximation of the temperature field at infinity, we have to obtain an asymptotic expansion of $T$ for the integration of Eq. (23). To determine the asymptotic behavior of $T$ at $r \gg 1$, the analytical result of outer temperature field in the continuous phase at the small parameter $\epsilon=1 / \sqrt{V_{\infty} M a}$ is given ${ }^{6}$ as

$$
T=r \cos \theta+\int_{\infty}^{r}(v \sin \theta-u \cos \theta-1) /\left.u\right|_{\Psi} d \tilde{r}+o(1)
$$

where $\Psi\left[=\frac{1}{2} \sin ^{2} \theta\left(r^{2}-1 / r\right)\right]$ is the streamfunction of the continuous phase and the symbol " + " before the integral is determined to preserve the monotonously increasing trend of $T(r, 0)$ with $r(>1)$ in the continuous phase. Using Eq. (38), it can be derived as

$$
T=r \cos \theta+\left.\int_{\infty}^{r} \frac{1}{\tilde{r}^{3}-1} \frac{1-3 \sin ^{2} \theta}{2 \cos \theta}\right|_{\Psi} d \tilde{r}+o(1) .
$$

Replacing $\theta$ by $\Psi$ in Eq. (41), we have

$$
T=r \cos \theta+\left.\int_{r}^{\infty} \frac{1}{\tilde{r}^{3}-1} \frac{3 \Psi /\left(\tilde{r}^{2}-1 / \tilde{r}\right)-1}{ \pm \sqrt{1-2 \Psi /\left(\tilde{r}^{2}-1 / \tilde{r}\right)}}\right|_{\Psi} d \tilde{r}+o(1),
$$

where the symbol " \pm " in the integral depends on the value of $\theta$ (the symbol " $+" /$ " $"$ " corresponds to $\theta \in[0, \pi / 2) /[\pi / 2, \pi))$. At $r \gg 1$, the result (42) can be expressed as

$$
\begin{aligned}
T & \approx r \cos \theta+\left.\int_{r}^{\infty} \frac{1}{\tilde{r}^{3}} \frac{3 \Psi / \tilde{r}^{2}-1}{ \pm \sqrt{1-2 \Psi / \tilde{r}^{2}}}\right|_{\Psi} d \tilde{r}+o(1) \\
& =r \cos \theta-\frac{1}{2 r^{2}} \cos \theta+o(1),
\end{aligned}
$$

where $\Psi \approx \frac{1}{2} \sin ^{2} \theta r^{2}$. derive

Using the temperature field at the infinity in (43), we can simplify Eq. (23) and Eq. (24) and

$$
\begin{aligned}
\left.\int_{0}^{\pi} \frac{\partial T}{\partial n}\right|_{r_{0}} r_{0}^{2} \sin \theta d \theta & =-\frac{1}{3 \epsilon^{2}}\left(1-\frac{1}{r_{\infty}^{3}}\right)+\frac{1}{\epsilon^{2}} \int_{0}^{\pi} f \sin \theta d \theta+o\left(\frac{1}{\epsilon^{2}}\right) \\
& \approx-\frac{1}{3 \epsilon^{2}}\left(1-3 \int_{0}^{\pi} f \sin \theta d \theta\right)
\end{aligned}
$$

and

$$
\left.\int_{0}^{\pi} \frac{\partial T^{\prime}}{\partial n}\right|_{r_{0}} r_{0}^{2} \sin \theta d \theta=\frac{1}{3 \lambda \epsilon^{2}}\left(2+3 \int_{0}^{\pi} f \sin \theta d \theta\right)
$$


From Eq. (44) and Eq. (45), we have

$$
\begin{aligned}
\int_{0}^{\pi}\left(\left.\beta \frac{\partial T^{\prime}}{\partial n}\right|_{r_{0}}-\left.\frac{\partial T}{\partial n}\right|_{r_{0}}\right) r_{0}^{2} \sin \theta d \theta & =\frac{1}{3 \epsilon^{2}}\left(1+\frac{2 \beta}{\lambda}\right)+\left(\frac{\beta}{\lambda}-1\right) \frac{1}{\epsilon^{2}} \int_{0}^{\pi} f \sin \theta d \theta \\
& =\frac{1}{3}\left(1+\frac{2 \beta}{\lambda}\right) V_{\infty} M a+\left(\frac{\beta}{\lambda}-1\right) V_{\infty} M a \int_{0}^{\pi} f \sin \theta d \theta .
\end{aligned}
$$

Since both $\beta$ and $\lambda$ are positive and $f \ll 1$, we have

$$
\left.\left.\beta \int_{0}^{\pi} \frac{\partial T^{\prime}}{\partial n}\right|_{r_{0}} r_{0}^{2} \sin \theta d \theta \gg \int_{0}^{\pi} \frac{\partial T}{\partial n}\right|_{r_{0}} r_{0}^{2} \sin \theta d \theta .
$$

So, if the overall steady-state energy with two phases in the flow domain under integral boundary conditions is balanced, Eq. (47) should be reduced to Eq. (26), which seems impossible. It is termed as a nonconservative integral thermal flux across the surface for the steady thermocapillary drop migration at large $M a(R e)$ numbers. This implies the overall steady-state energy unbalance of two phases in the flow domain in the co-moving frame of reference and indicates that the thermocapillary drop migration at large $M a(R e)$ numbers cannot reach steady state. Thus, it is clear that the invalid assumption of quasi-steady state for the thermocapillary drop migration process is a reasonable explanation for the nonconservative integral thermal flux across the drop surface.

To analyze the thermal flux near the boundary, we write the integrals of Eq. (44) and Eq. (45) in the discretization scheme as follows

$$
\left.\int_{0}^{\pi} \frac{\partial T}{\partial n}\right|_{r_{0}} r_{0}^{2} \sin \theta d \theta=\sum_{i=1}^{N} \frac{\partial T}{\partial n}\left(r_{0}, \theta_{i}\right) r_{0}^{2} \sin \theta_{i} \Delta \theta<0
$$

and

$$
\left.\int_{0}^{\pi} \frac{\partial T^{\prime}}{\partial n}\right|_{r_{0}} r_{0}^{2} \sin \theta d \theta=\sum_{i=1}^{N} \frac{\partial T^{\prime}}{\partial n}\left(r_{0}, \theta_{i}\right) r_{0}^{2} \sin \theta_{i} \Delta \theta>0,
$$

where $\theta_{i} \in[0, \pi]$ and $\Delta \theta=\pi / N$. Since $r_{0}^{2} \sin \theta_{i} \geq 0$, we reach a conclusion that there must be some interface points $\theta_{i} \in[0, \pi]$ where the following equation holds

$$
\frac{\partial T}{\partial n}\left(r_{0}, \theta_{i}\right)<0<\frac{\partial T^{\prime}}{\partial n}\left(r_{0}, \theta_{i}\right),
$$

or some interface points $\theta_{i}$ and $\theta_{j} \in[0, \pi]$ where the following equations hold

$$
\begin{aligned}
& 0<\frac{\partial T}{\partial n}\left(r_{0}, \theta_{i}\right)<\frac{\partial T^{\prime}}{\partial n}\left(r_{0}, \theta_{i}\right), \\
& \frac{\partial T}{\partial n}\left(r_{0}, \theta_{j}\right)<\frac{\partial T^{\prime}}{\partial n}\left(r_{0}, \theta_{j}\right)<0 .
\end{aligned}
$$

Physically, this means that near these points $\theta_{i}$ the thermal energy is transferred from the interface to outside (the surrounding fluid) as well as from the interface to inside (the droplet) or near these points $\theta_{i} / \theta_{j}$ the transference of thermal energy from outside/the interface to the interface/outside is weaker/stronger than that from the interface/inside to inside/the interface. On the one hand, if Eq. (50) can satisfy the integral thermal flux boundary condition in Eq. (26), thermal sources inside the interface will be introduced to balance the transference of thermal energy. On the other hand, if Eq. (51) can satisfy the integral thermal flux boundary condition in Eq. (26), thermal sinks inside the interface or thermal sources in the droplet will be introduced to decrease the transference of thermal energy from the interface to outside or to increase the transference of thermal energy from inside to the interface. Since there is absolutely no thermal sources or sinks inside the interface or thermal sources in the droplet, the above transport processes of thermal energy near the interface seem impossible. It means that the thermal flux across the drop surface is nonconservative.

\section{CONCLUSIONS}

In summary, from the condition of overall steady-state energy balance with two phases in a flow domain, we have identified a conservative/nonconservative integral thermal flux across the surface for a steady thermocapillary migration of a drop with a slight axisymmetric deformation from a 
sphere in a uniform temperature gradient at small/large $M a(R e)$ numbers. The conservative integral thermal flux confirms the assumption of quasi-steady state in thermocapillary drop migration at small $M a(R e)$ numbers. The nonconservative integral thermal flux may well result from the invalid assumption of quasi-steady state, which indicates that the thermocapillary drop migration at large $M a(R e)$ numbers cannot reach steady state and is thus a unsteady process.

\section{ACKNOWLEDGMENTS}

One of the authors (Z.-B.W.) thanks the National Science Foundation for general support (under Grant No. 11172310) and IMECH/SCCAS SHENTENG 1800/7000 research computing facilities for assisting in the computation.

\section{APPENDIX: ENERGY EQUATIONS UNDER TRANSFORMATION BETWEEN TWO INERTIA FRAMES}

Based on the transformation between two cylindrical coordinate systems $(\bar{r}, \bar{z})$ and $(r, z)$ of Eq. (4), we have

$$
\begin{aligned}
& \left.\bar{\nabla}\right|_{t}=\left.\frac{\partial}{\partial \bar{r}}\right|_{t} \mathbf{i}+\left.\frac{\partial}{\partial \bar{z}}\right|_{t} \mathbf{k}=\left.\frac{\partial}{\partial r}\right|_{t} \mathbf{i}+\left.\frac{\partial}{\partial z}\right|_{t} \mathbf{k}=\left.\nabla\right|_{t}, \\
& \left.\bar{\Delta}\right|_{t}=\left.\frac{1}{\bar{r}}\left[\frac{\partial}{\partial \bar{r}}\left(\bar{r} \frac{\partial}{\partial \bar{r}}\right)+\frac{\partial}{\partial \bar{z}}\left(\bar{r} \frac{\partial}{\partial \bar{z}}\right)\right]\right|_{t}=\left.\frac{1}{r}\left[\frac{\partial}{\partial r}\left(r \frac{\partial}{\partial r}\right)+\frac{\partial}{\partial z}\left(r \frac{\partial}{\partial z}\right)\right]\right|_{t}=\left.\Delta\right|_{t} .
\end{aligned}
$$

And for energy equation (1) of the continuous phase fluid, we can also write its unsteady, convection and conductivity terms as follows

$$
\begin{aligned}
\left.\frac{\partial \bar{T}}{\partial t}\right|_{\overline{\mathbf{r}}} & =\left.\frac{\partial T}{\partial t}\right|_{\overline{\mathbf{r}}}+G V_{\infty}=\left.\left.\frac{\partial T}{\partial r}\right|_{t} \frac{\partial r}{\partial t}\right|_{\overline{\mathbf{r}}}+\left.\left.\frac{\partial T}{\partial z}\right|_{t} \frac{\partial z}{\partial t}\right|_{\overline{\mathbf{r}}}+\left.\left.\frac{\partial T}{\partial t}\right|_{\mathbf{r}} \frac{\partial t}{\partial t}\right|_{\overline{\mathbf{r}}}+G V_{\infty} \\
& =\left.\frac{\partial T}{\partial z}\right|_{t}\left(-V_{\infty}\right)+\left.\frac{\partial T}{\partial t}\right|_{\mathbf{r}}+G V_{\infty}=-V_{\infty} \frac{\partial T}{\partial z}+G V_{\infty}, \\
\left.\overline{\mathbf{v}} \bar{\nabla} \bar{T}\right|_{t} & =\left.\left(\mathbf{v}+V_{\infty} \mathbf{k}\right) \bar{\nabla}\left(T+G V_{\infty} t\right)\right|_{t}=\left.\left(\mathbf{v}+V_{\infty} \mathbf{k}\right) \bar{\nabla} T\right|_{t} \\
& =\mathbf{v} \nabla T+V_{\infty} \frac{\partial T}{\partial z}, \\
\left.\bar{\Delta} \bar{T}\right|_{t} & =\left.\bar{\Delta}\left(T+G V_{\infty} t\right)\right|_{t}=\left.\bar{\Delta} T\right|_{t}=\Delta T,
\end{aligned}
$$

where $\left.\frac{\partial r}{\partial t}\right|_{\overline{\mathbf{r}}}=\left.\frac{\partial r}{\partial t}\right|_{\bar{r}}=0,\left.\frac{\partial z}{\partial t}\right|_{\overline{\mathbf{r}}}=\left.\frac{\partial z}{\partial t}\right|_{\bar{z}}=-V_{\infty}$, and $\left.\frac{\partial T}{\partial t}\right|_{\mathbf{r}}=0$. Then, substituting Eq. (A2) into Eq. (1) in the laboratory coordinate system, we obtain Eq. (5) in a coordinate system with its origin fixed at the drop center

$$
G V_{\infty}+\mathbf{v} \nabla T=\kappa \Delta T
$$

Similarly, we can also transform the energy equation within the drop as above.

${ }^{1}$ R. Monti, Physics of Fluids in Microgravity (CRC, 2001).

${ }^{2}$ N. O. Young, J. S. Goldstein, and M. J. Block, "The motion of bubbles in a vertical temperature gradient," J. Fluid Mech. 6, 350 (1959).

${ }^{3}$ R. S. Subramanian, "Slow migration of a gas bubble in a thermal gradient," AIChE J. 27, 646 (1981).

${ }^{4}$ A. Crespo, E. Migoya, and F. Manuel, "Thermocapillary migration of bubbles at large Reynolds numbers," Int. J. Multiphase Flow 24, 685 (1998).

${ }^{5}$ A. Crespo and J. Jimenez-Fernandez, "Thermocapillary migration of bubbles at moderately large Reynolds numbers," in Microgravity Fluid Mechanics, edited by H. J. Rath (Springer, Berlin, 1992), p. 405.

${ }^{6}$ R. Balasubramaniam and R. S. Subramanian, "Thermocapillary bubble migration- thermal boundary layers for large Marangoni numbers," Int. J. Multiphase Flow 22, 593 (1996).

${ }^{7}$ R. Balasubramaniam and J. E. Lavery, "Numerical simulation of thermocapillary bubble migration under microgravity for large Reynolds and Marangoni numbers," Numer. Heat Transfer, A 16, 175 (1989).

${ }^{8}$ M. Treuner, V. Galindo, G. Gerbeth, D. Langbein, and H. J. Rath, "Thermocapillary bubble migration at high Reynolds and Marangoni numbers under low gravity,” J. Colloid Interface Sci. 179, 114 (1996).

${ }^{9}$ P. H. Hadland, R. Balasubramaniam, G. Wozniak, and R. S. Subramanian, "Thermocapillary migration of bubbles and drops at moderate to large Marangoni number and moderate Reynolds number in reduced gravity," Exp. Fluids 26, 240 (1999).

${ }^{10}$ B. Braun, C. Ikier, and H. Klein, "Thermocapillary migration of droplets in a binary mixture with miscibility gap during liquid/liquid phase separation under reduced gravity,” J. Colloid Interface Sci. 159, 515 (1993). 
${ }^{11}$ G. Wozniak, "On the thermocapillary motion of droplets under reduced gravity," J. Colloid Interface Sci. 141, 245 (1991).

${ }^{12}$ J. C. Xie, H. Lin, P. Zhang, F. Liu, and W. R. Hu, "Experimental investigation on thermocapillary drop migration at large Marangoni number in reduced gravity," J. Colloid Interface Sci. 285, 737 (2005).

${ }^{13}$ R. Balasubramaniam and R. S. Subramanian, "The migration of a drop in a uniform temperature gradient at large Marangoni numbers," Phys. Fluids 12, 733 (2000).

${ }^{14}$ X. Ma, R. Balasubramiam, and R. S. Subramanian, "Numerical simulation of thermocapillary drop motion with internal circulation," Numer. Heat Transfer A 35, 291 (1999).

${ }^{15}$ M. Herrmann, J. M. Lopez, P. Brady, and M. Raessi, "Thermocapillary motion of deformable drops and bubbles," in Proceedings of the Summer Program 2008 (Center for Turbulence Research, Stanford University, 2008), p. 155.

${ }^{16}$ Z.-B. Wu and W. R. Hu, "Thermocapillary migration of a planar droplet at moderate and large Marangoni numbers," Acta Mech. 223(3), 609 (2012).

${ }^{17}$ R. Balasubramaniam and A. Chai, "Thermocapillary migration of droplets: An exact solution for small Marangoni numbers," J. Colloid and Interface Sci. 119, 531 (1987).

${ }^{18}$ M. D. Levan, "Motion of a droplet with a Newtonian interface," J. Colloid Interface Sci. 83, 11 (1981).

${ }^{19}$ R. S. Subramanian, "Thermocapillary migration of bubbles and droplets," Adv. Space Res. 3, 145 (1983).

${ }^{20}$ J. F. Happer and D. W. Moore, "The motion of a spherical liquid drop at high Reynolds number," J. Fluid Mech. 32, 367 (1968). 